Revue d'histoire de l'Amérique française

QREVUE D.HISTOIRE DE L'AMÉRIQUE FRANÇAISE

\title{
Le régime seigneurial au pays de Québec, 1760-1854 (1er article)
}

\section{Maurice Séguin}

Volume 1, numéro 3, décembre 1947

URI : https://id.erudit.org/iderudit/801387ar

DOI : https://doi.org/10.7202/801387ar

Aller au sommaire du numéro

Éditeur(s)

Institut d'histoire de l'Amérique française

ISSN

0035-2357 (imprimé)

1492-1383 (numérique)

Découvrir la revue

Citer cet article

Séguin, M. (1947). Le régime seigneurial au pays de Québec, 1760-1854 (1er article). Revue d'histoire de l'Amérique française, 1(3), 382-402.

https://doi.org/10.7202/801387ar d'utilisation que vous pouvez consulter en ligne.

https://apropos.erudit.org/fr/usagers/politique-dutilisation/ 


\title{
LE REGIME SEIGNEURIAL AU PAYS DE QUEBEC, 1760-1854
}

\author{
ler article
}

\section{MAINTIEN DU RÉGIME}

On distingue d'abord une phase passive. Rien dans les Capitulations n'abolit la tenure seigneuriale. Les cadres du régime ne sont pas disloqués par la Conquête: la plupart des seigneurs restent au pays; ceux qui partent sont remplacés par des Britanniques. D'ailleurs, avant le Traité de 1763, on vit dans le provisoire. En 1762, Murray trouve tout naturel de concéder d'une façon qui équivaut au mode seigneurial ${ }^{1}$.

La Proclamation Royale (1763) et les Instructions ordonnent de concéder à l'avenir en franc et commun soccage et imposent les lois anglaises sur la propriété ${ }^{2}$. C'était prohiber l'extension du régime seigneurial et donner aux seigneuries déjà existantes un caractère britannique. Avec le retour aux lois françaises, l'obligation subsistait encore de concéder en soccage les terres de la Couronne.

Mais on passe à la phase active. L'autorité ne se contente pas de conserver les seigneuries déjà tracées, elle veut en concéder de nouvelles. Carleton est convaincu des avantages du régime; il y voit un organisme propre à entretenir le loyalisme des nouveaux sujets, liant les censitaires aux seigneurs et les seigneurs au Roi. A la demande du

1. W. B. Munro, The Seigniorial System in Canada, New York 1907, 189-203.I. Caron, La colonisation de la Province de Québec, 1760-1791, Québec 1923, Appendice III.

2. The Royal Proclamation, 7 octobre 1763, dans W. P. M. Kennedy, Statutes, Treaties and Documents of the Canadian Constitution, 1713-1929, Toronto 1930, 36-7. Instructions à Murray, 7 décembre 1763, dans A. Short et A. G. Doughty, Documents relatifs à l'histoire constitutionnelle du Canada, 1759-1791, Ottawa 1921, 155. (Ces doc. désormais cités sous le sigle DRHC). 
gouverneur, Londres consent, en 1771 , à ce que les terres soient à l'avenir concédées exclusivement selon la tenure seigneuriale ${ }^{3}$. C'est alors que des gens importants de la colonie, une quarantaine de Britanniques et quelques Canadiens, pétitionnent pour se faire octroyer des seigneuries ${ }^{4}$. Si l'on voulait spéculer sur les terres, c'était le seul moyen présumé efficace pour attirer des colons; car selon l'opinion courante de l'époque, le Nord serait toujours peuplé uniquement de Canadiens, sauf dans les villes. L'Acte de Québec (1774) confirme l'ordre de 1771. Carleton n'a pas voulu qu'on y insérât une clause pourvoyant à la mutation volontaire des tenures parce que la colonie ne le réclamait pas. Et le gouverneur avertit que, si plus tard on permet cette mutation, il faudra user de précautions car l'abolition du régime seigneurial aff ranchirait les seigneurs de leurs devoirs envers la Couronne ${ }^{5}$.

En 1775, les armées des Révoltés envahissent le Québec. L'occasion se présente d'éprouver l'utilité du régime seigneurial. Carleton ordonne une levée de censitaires par l'entremise desseigneurs. Beaucoup de seigneurs font preuve de zèle; mais la masse des censitaires récalcitre $^{6}$. Grande désillusion pour les autorités! On a voulu voir dans cet incident, la preuve du peu d'attachement des censitaires au régime; quinze ans de " liberté britannique " auraient suffi pour leur révéler tout l'odieux de l'asservissement féodal ${ }^{7}$. Ne serait-ce pas plutôt que les Canadiens refusajent de se battre pour l'étranger, par désir de rester neutres dans cette querelle intestine entre gens de langue anglaise et afin d'éviter de se faire massacrer à peine quinze ans après les dévastations des luttes suprêmes? D'ailleurs, ce n'était pas le premier exemple de conscription mal accueillie; déjà en 1764, Murray

3. Carleton à Shelburne, 12 avril 1768, APC, Q5-2: 477. Instructions additionnelles à Carleton, 2 juillet 1771, dans Shortt-Doughty, DRHC, 1759-1791, 401.

4. Pétitions au Comité des Terres, 9 octobre 1772, 7 octobre 1773, 11 novembre 1774, APC, Q8:206-12; Q9:131-50; Q11:2-8.

5. Troisième projet de loi. Objections de Hillsborough contre le Bill de Québec, dans Shortt-Doughty, DRHC, 1759-1791, 528-37. Instructions à Carleton, 3 janvier 1775, ibid., 578.

6. Cramahé à Dartmouth, Carleton à Dartmouth, 21 septembre 1775, APC, Q11:249, 261.

7. G. S. Graham, British Policy and Canada, 1774-1791, Londres 1930, 28-30. 
et Haldimand avaient dâ recourir aux menaces pour lever quelques volontaires contre les Indiens ${ }^{8}$.

L'aventure de 1775 porta un rude coup au prestige des seigneurs. Mais les Canadiens étaient-ils prêts pour autant à délaisser le mode seigneurial d'acquérir de la terre, au profit d'une tenure inconnue et étrangère. Rien ne l'indique. 1775 ne prouve qu'une chose: le régime a failli comme instrument de conscription. Cependant les autorités ne perdent pas encore tout espoir en son utilité. Il peut servir le pouvoir par le maintien d'une classe d'aristocrates; certains seigneurs zélés ne viennent-ils pas de le démontrer?

\section{L'ARRIVÉE DES LOYAL!STES}

Les Loyalistes arrivent dans une colonie où l'on ne peut concéder que selon le mode seigneurial. On tente de les acclimater au régime. Haldimand reçoit l'ordre de leur arpenter, autour de Cataracoui, des seigneuries relevant directement du Roi ${ }^{9}$. Les Loyalistes protestent, menacent d'abandonner plutôt le pays et exigent un district spécial ${ }^{10}$. Les marchands britanniques de Québec les appuient ${ }^{11}$. Le représentant des Loyalistes au Conseil va même jusqu'à prôner la conversion des tenures pour rendre toute la colonie "semblable aux autres "12. La situation est tellement changée, que Dorchester, cette fois-ci, demande la permission de concéder en soccage. Londres la lui accorde avec empressement dès octobre $1787 .{ }^{13}$ On crée de nouveaux districts à l'intérieur de l'immense Québec. Les colons britanniques s'orientent vers le " séparatisme ») et vers le morcellement de l'Amérique du Nord

8. Murray aux capitaines de milice des paroisses au-dessus de Québec, 22 mars 1764, RAPC, (1917-18), 21. Haldimand, Ordre aux capitaines de milice... 8 mars 1764, ibid., 152.

9. In structions additionnelles à Haldimand, 16 juillet 1783, dans Shortt-Doughty DRHC, 1759-1791, 721.

10. Pétition des Loyalistes au Roi, avril 1785, APC, Q24-1:77-8.

11. Rapport des Marchands de Québec au comité du Conseil, 5 janvier 1787, APC, Q27-1:260.

12. J. Johnson, en dissidence au rapport du comité du Conseil concernant la population, l'agriculture et la colonisation, 13 février 1787, APC, Q27-2:502.

13. Dorchester à Sydney, 13 juin 1787, APC, Q27-2:923. Instructions à Dorchester, 26 octobre 1787, APC, Q28:377. 
britannique en plusieurs provinces. Et voilà le régime seigneurial parmi les causes qui les éloignent des Canadiens et les empêchent de fonder leurs premiers centres de colonisation agricole au milieu de ceux-ci.

Le Haut-Canada n'aura pas de seigneuries. Mais que faire de celles du Bas-Canada? C'est alors que se déclenche l'attaque de 1790 contre le régime seigneurial. Comme en 1774 , le projet de la future constitution comporte une clause pour légaliser le changement de tenure. Dorchester, cette fois, acquiesce et charge son Conseil d'enquêter ${ }^{14}$. Celui-ci ressuscite une pétition signée en 1788, par un de ses membres, Charles de Lanaudière. Ce seigneur voudrait attirer dans ses fiefs à peine peuplés des Loyalistes et d'autres immigrants britanniques; c'est surtout par eux, estime-t-il, que dorénavant la colonie se développera. Ma:s ces gens ont en horreur la tenure seigneuriale; de Lanaudière demande donc que ses fiefs soient repris par la Couronne et qu'ils lui soient reconcédés en franc et commun soccage ${ }^{15}$. La majorité du Conseil approuve cette pétition, attribue la lenteur du développement du Québec au régime seigneurial et s'apitoie sur le sort des seigneurs condamnés à ne pouvoir peupler leurs seigneuries que lorsque les cantons qu'on se propose de fonder, seront tous d'abord colonisés. Le Conseil se prononce en faveur d'une mutation de tenure, non pas obligatoire et générale, mais volontaire. Un conseiller (britannique pourtant) se fait le défenseur du régime seigneurial et des censitaires canadiens. La mutation, soutient Mabane, rendrait difficile l'établissement des enfants des familles nombreuses; elle mettrait les cultivateurs à la merci du seigneur, rendrait ce dernier propriétaire absolu des terres vacantes, libre d'augmenter les taux de concession ou de vendre, alors que, sous le régime seigneurial, les charges sont peu nombreuses, déterminées, nullement oppressives ${ }^{17}$. Un autre vigoureux plaidoyer en faveur du régime et une pétition signée à la

14. Grenville à Dorchester, 20 octobre 1789, APC, Q42:96. Dorchester à Grenville, 8 février, 25 septembre 1790, APC, Q41-1:20, Q46-1:2.

15. Pétition de C. de Lanaudière à Dorchester, 26 janvier 1788, APC, Q48-1:5.

16. Résolutions du Conseil, 11 octobre 1790, APC, Q48-1:17.

17. A. Mabane, en dissidence aux résolutions du Conseil, 15 octobre 1790, APC, Q48-1:27-31. 
fois par des seigneurs et des censitaires viennent appuyer Mabane ${ }^{18}$. Ici il convient de souligner le beau désintéressement des seigneurs de n'avoir pas écouté leurs seuls intérêts matériels; tous, en effet, adversaires comme tenants de la mutation reconnaissaient que celle-ci favoriserait les seigneurs et les Britanniques et ne pourrait s'opérer qu'au détriment des paysans canadiens. Encore une fois, la clause pourvoyant à la conversion des tenures fut rayée de la loi projetée. Comment expliquer ce dénouement? Par ce fait peut-être que, dans le Québec, en dehors du territoire occupé par les seigneuries, l'espace ne manquait pas. De ce côté les Britanniques allaient désormais porter leurs efforts.

On avait en 1771 autorisé la concession de nouvelles seigneuries. Encore en état de coloniser à l'aise dans les anciennes, les Canadiens ne se préoccupèrent pas d'en demander de nouvelles. Au reste, les pétitions des Britanniques et de quelques Canadiens pour l'obtention des seigneuries vers 1772-1774 n'aboutirent pas, pour cette double raison que toutes concessions furent ajournées durant les hostilités (1774-1783), et que l'après-guerre offrait aux spéculateurs des perspectives plus avantageuses du côté des cantons. On ne retrace qu'une seule concession de seigneurie: en 1788, sur la Baie des Chaleurs, loin des établissements canadiens ${ }^{19}$. Ainsi, alors que permission était donnée de créer de nouvelles seigneuries, les Canadiens laissèrent passer l'occasion sans s'en prévaloir. Désintéressement funeste qui aidera les autorités à supprimer la méthode seigneuriale dans la concession des terres vacantes de la Couronne.

La constitution de 1791 décrète obligatoire, dans le Haut-Canada, la concesssion en franc et commun soccage, et dans le Bas-Canada, décrète le même mode de concession, facultatif, au gré du concessionnaire ${ }^{20}$. La priorité paraissait donc appartenir, dans le Bas-Canada, à la tenure seigneuriale; à moins de demande contraire des individus, et pour des cas particuliers, il semblait que les autorités s'y confor-

18. M. Bédard, ptre, Observations sur le Projet du Changement de Tenure, 16 février 1791, APC, Q51-2:506. Pétition à Dorchester, contre le changement de Tenures, 24 mai 1791, APC, Q51-2:454.

19. Minutes du Comité des Terres, juin 1788, seigneurie concédée à J. Shoolbred, APC, Q37:252-62.

20. Article XLIII de l'Acte constitutionnel (1791) dans Shortt-Doughty, DRHC, 1759-1791, 1029. 
meraient comme à la pratique courante. Ce n'était que camouflage. Les instructions dont s'accompagne la loi de 1791 sont identiques sur ce point pour les deux Canadas; les administrateurs coloniaux reçoivent l'ordre de n'organiser que des concessions en soccage ${ }^{21}$. Loin de se tenir prêtes à concéder des seigneuries, les autorités du Bas-Canada prennent les devants, annoncent, dès février 1792, la division des terres de la Couronne en " townships " et se lancent dans une vaste distribution de concessions en soccage ${ }^{2}$. Encore cette fois, les Canadiens, toujours à l'aise dans les vieilles seigneuries, ne protestent pas. La concession de nouvelles seigneuries était donc délibérément écartée et, comme on le verra par la suite, pratiqusment abolie dès 1791-1792.

\section{R FORME OU ABOI.ITION?}

Les anciennes seigneuries subsistaient. " Mais la protection du roi français, reconnaissait le juge Monk en 1794, ne fut pas maintenue et les pouvoirs du gouvernement français ne furent pas rétablis ou ne purent être exercés "). ${ }^{23}$ Surtout l'intəndant n'était plus là pour garder tout le système en bon ordre. Certains seigneurs augmentèrent les charges au détriment de leurs censitaires: fait admis par les plus ardents défenseurs du régime ${ }^{24}$.

Une des premières protestations vint des censitaires de Longueuil en $1793^{25}$. L'autorité se montra favorable aux censitaires; les seigneurs, affirmèrent le gouverneur, et les hauts fonctionnaires, n'avaient pas le droit d'augmenter les taux de concession ${ }^{26}$. L'Assəmblée voulut en-

21. Instructions à Dorchester, 7 septembre 1791, dans A. G. Doughty et D. A. Mc Arthur, Documents relatifs d̀ l'histoire constitutionnelle du Canada, 1791-1818, Ottawa 1915, 13.

22. A. Clarke, Proclamation à ceux qui désirent s'établir sur les Terres de la Couronne dans la Province du Bas-Canada, 16 février 1792, ibid., 61.

23. Monk à Dundas, 6 juin 1794, APC, Q69-2: 261.

24. M. Bédard ptre, Observations sur le Projet du Changement de Tenures, 16 février 1791, APC Q51-2: 527.

25. Requête des censitaires de Longueuil, à la Chambre d'Assemblée, mars 1793, APC, Q67:79.

26. Dorchester à Dundas, 24 février 1794, APC, Q67:77. Sewell à Dorchester, 24 février 1794, APC, Q67:88. Dundas à Dorchester, 5 juillet 1794, APC, Q67:175. 
quêter et obliger les seigneurs à concéder aux anciens taux. Seulement il arriva que des seigneurs (britanniques surtout) voulurent profiter de l'occasion, semble-t-il, pour obtenir l'abolition du régime seigneurial; ils se prirent à le dénoncer comme suranné эt oppressif, dans l'espoir au fond de devenir maîtres absolus de leurs domaines, et pour y établir avec profit des colons anglo-saxons. Ce double jeu, mis à jour, fit suspendre l'enquête et rien n'aboutit ${ }^{27}$. Par la suite, les cours de la colonie donneront raison aux seigneurs; l'Assemblé aura des velléités de remédier; il arrive encore que, durant la période, des plaintes se renouvellent contre "quelques" seigneurs.

Ainsi, deux courants se dessinent et qu'il importe de ne pas confondre: d'une part les attaques des Canadiens contre les abus en vue de réformer le régime; d'autre part les attaques des Britanniques contre le régime même afin d'obtenir son abolition.

$\mathrm{Au}$ moment où l'Assemblée législative du Bas-Canada donne ses premiers signes d'indocilité, Milnes se rend compte de la faillite du rêve de Carleton qui espérait faire des seigneurs des aristocrat ss au service du pouvoir. Les seignəurs auraient dû maintenir la loyauté envers le gouvernement, servir de frein et de contrepoids dans l'Assemblée coloniale; or ces seigneurs sont pauvres, reçoivent très peu de leurs censitaires et restent sans influence sur ceux-ci ${ }^{28}$. Pour les Britanniques, le régime seigneurial apparaissait donc inutile et au point de vue militaire et au point de vue administratif. Après le déboire de 1775, venait celui de 1800 .

Le régime s'avérait même nuisible. Quarante ans se sont écoulés depuis la Conquête; et le Bas-Canada, on le constate avec peine, est resté français. Une politiquə avisée commandait də mêler Britanniques ot Canadiens afin d'assimiler ces derniers; or le régime seigneurial abhorré par les colons britanniques constitue à la fois et l'obstacle au peuplement des campagnes par ces derniers, et par suite, l'obstacle à l'anglicisation des Canadiens. Une conclusion s'impose: abolir le régime non par suppression directe, par crainte des préjugés des Canadiens, mais d'une façon indirecte et graduelle. ${ }^{29} \mathrm{Et}$ voici alors que

27. F.-X. Garneau, Histoire du Canada, Montréal 1945, 7:36-7.

28. Milnes à Portland, 1 novembre 1800, APC, Q95:228. 175.

29. J. Sewell, Rapport sur le Bill de lods et ventes, 4 avril 1801, APC, Q86-1: 
s'esquisse la deuxième attaque contre le régime, celle de 1801. Depuis 1763 , les nouveaux administrateurs de la colonie ont négligé de percevoir des censitaires directs du Roi, surtout des bourgeois de la ville də Québec, une taxe seigneuriale appelée lods et ventes, taxe assez onéreuse qui pouvait s'élever jusqu'à un douzième de la valeur de la propriété, chaque fois qu'une transaction équivalait à une vente. Une telle réclamation, pensait-on, provoquerait l'indignation des censitaires (dont plusieurs étaient des marchands britanniques); elle les inciterait à exiger la suppression de la tenure seigneuriale dans la capitale du Bas-Canada. Et de là pouvait venir, du moins on l'escomptait, le prélude d'une mutation genérale des tenures. ${ }^{30}$. Ces espoirs ne se réaliseront pas. Retenons cependant que par cette question de lods et ventes on venait de s'attaquer à un point névralgique: le commerce.

Au temps de la crise politique sous Craig, l'antienne revient d'un peuplement expéditif et à tout prix du Bas-Canada au moyen de colons anglo-saxons, et voire américains. Hélas, le régime seigneurial est là qui " inspire une aversion profonde à tous les Anglais et à tous les Américains "). Une loi s'imposerait pour la conversion des tenures; mais, cette loi, comment l'attendre de l'actuelle Assemblée ${ }^{31}$ ?

On n'eut pas besoin de " républicains ". Après 1815, la GrandeBretagne se délesta d'une forte émigration. La chance s'offrait de britanniser enfin le Bas-Canada. Un seigneur dirige alors la troisième attaque contre le régims seigneurial. En 1816, J. Caldwell, possesseur de plusieurs seigneuries peu peuplées, désire en transformer la tenure pour attirer des immigrants ${ }^{32}$. Les autorités accueillent cette demande avec empressement ${ }^{3}{ }^{3}$. Mais deux obstacles se dressent: recourra-t-on a l'Assomblée hostile afin de fixer une compensation pour le droit de quint que la Couronne abandonnerait? Faudra-t-il tailler des réserves du clergé dans les seigneuries reconcédées en franc et commun soccage ? $^{34}$

30. Milnes à Portland, 16 avril, 10 juin 1801, APC, Q86-1:157, Q87-1:93.

31. J. Sewell, Mémoire inclus dans Craig à Liverpool, 1er mai 1810, APC, Q112: 196.

32. Caldwell à Bathurst, 5 avril 1816, APC, G8:210.

33. Bathurst à Drummond, 4 mai 1816, APC, G8:206.

34. Correspondance échangée entre Bathurst et Sherbrooke et Rapports des jurisconsultes, 1816-17, dans Doughty-McArthur, DRHC, 1791-1818, 497-505. 
Ces deux points nécessiteront l'intervention du parlement impérial. Il en résultera un retard de cinq ans.

\section{DEMANDE DE NOUVELLES SEIGNEURIES}

Vers 1820 , on croit distinguer un ralentissement dans la colonisation agricole des Canadiens et la Chambre enquête pour en découvrir les causes. On donne comme première raison de ce ralentissement que, dans les seigneuries des régions les plus recherchées, les terres propres à la culture sont presque toutes occupées. On ajoute aussi que " certains ") seigneurs haussent les taux de concession et vendent des lots; on demande la réforme de ces abus ${ }^{35}$. Quant aux terres de la Couronne, une des principales causes qui en retardent la colonisation est " l'abandon de l'ancienne méthode de concéder en fief et seigneuria et l'introduction de la tenure en franc et commun soccage $)^{36}$. Sans se prononcer sur les avantages respectifs des deux tenures pour un pays plus avancé, le comité de l'Assemblée chargé de l'enquête entreprend une justification économique du régime seigneurial pour la colonie dans l'état actuel:

Les deux grands obstacles à la colonisation dans tout jeune pays sont le manque de capital et par suite le manque de débouché pour le travail

Le système le moins onéreux pour le colon est sans contredit le système français des Seigneuries.

Cette tenure offre au colon plusieurs avantages: celui-ci n'est obligé de débourser aucune somme d'argent pour sa terre; le capital qu'il possède, si maigre soit-il, peut être entièrement employé à améliorer sa ferme ${ }^{37}$

Le comité souligne en outre d'autres raisons d'ordre religieux et social (besoin de prêtres, besoin de se grouper) et conclut que rien n'est plus apte à produire cet ensemble de conditions qu'exigent les Canadiens pour coloniser que " la création d'un nombre suffisant de

35. Lettres des Cures des Paroisses du Bas-Canada, Québec 1823, 30, 33, 51, 66.

36. First Report of the Committee of the House of Assembly (on the) Crown Lands, Quebec 1821, 4.

37. Ibid., 8-9. 
seigneuries pour fournir des habitations et des terres au surplus de population du pays ${ }^{38}$. ")

$\mathrm{Au}$ moment où les Britanniques projettent, avec plus d'ardeur et de raison que jamais, la conversion des tenures, voici que les Canadiens, sentant le sol seigneurial leur manquer, réclament la création de nouvelles seigneuries. Il ne faut pas perdre du vue un de leurs arguments: l'acquisition de la terre selon le mode seigneurial est ce qu'il y a de plus conforme à leur état de paysans sans capitaux ni débouchés pour leurs produits. Cet argument prend toute sa signification si on a soin de se souvenir qu'à ce moment (1820) une protestation massive allait s'élever de la part des colons (britanniques et canadiens) contre les corn laws. On ne trouvait même pas à vendre un faible surplus de blé.

\section{Inter vention du Parlement Impérial.}

Comme solutions aux deux problèmes de 1816, le parlement impérial votэ, en 1822, une loi qui donne droit aux seigneurs de remettre à la Couronne la partie vacante de leurs fiefs et de recevoir en retour ces mêmes terres en franc et commun soccage, moyennant une indemnité pour le droit de quint que la Couronne abandonne et sans qu'on soit obligé de retenir un septième des lots comme réserves du clerge ${ }^{3}{ }^{9}$. Cette loi était inofficace et il a fallu la compléter, a-t-on dit plus tard, parce qu'elle ne permettait pas aux censitaires de se débarrasser du régime, " alors que les personnes qui désiraient changer de tenure étaient les habitants bien plus que les seigneurs " ${ }^{40}$. Rien de plus erroné que cette interprétation. En 1820, les Canadiens réclamaient des seigneuries. A l'origine de la loi de 1822, on retrouve non pas des pétitions de censitaires canadiens, mais une pétition d'un seigneur britannique (Caldwell). Et ce ne sont pas des censitaires canadiens mais un autre seigneur britannique, (E. Ellice), qui poussera le gouvernement impérial à compléter sa législation de 1822, afin de libérer le Bas-Canada du régime seigneurial, " the greatest if not the only bar to the improvement and advancement of the Colony "); ce qui se

38. Ibid., 10.

39. 3 Geo IV, cap. 119, xxxi, xxxii, APC, Q163-1:1.

40. Munro, The Seigniorial System in Canada, 224-5. 
traduit par : le plus grand obstacle à l'anglicisation de la colonie et au peuplement de celle-ci par les immigrants britanniques ${ }^{4}$.

Papineau reconnaît que la loi de 1822 tire son origine de quelques individus hostiles et intér sssés; il ne voit pas en quoi néanmoins cette loi peut causer du tort aux Canadiens, puisque la terre seigneuriale propre à la culture n'est plus considérable. La conversion des tenures arriverait trop tard pour être vraiment utile aux Britanniques et leur permettre de coloniser les seigneuries; il n'y reste presque plus de place ${ }^{42}$. Cependant l'Assemblée croit devoir protester et demande que si un seigneur transforme la tenure de ses domaines vacants, il soit quand même tenu de concéder selon les taux ordinaires aux paysans qui désireront des $\operatorname{lots}^{43}$.

En 1825, autre loi impériale. Celle-ci décrète que le seigneur qui aura obtenu de la Couronne la conversion de tenure, pour ses fiefs, sera obligé d'accorder les mêmes avantages à ceux de ses censitaires qui voudront commuer ${ }^{44}$. Afin d'accélérer la disparition du régime, Londres fixe à $5 \%$ seulement de la valeur de la seigneurie, l'indemnité payable à la couronne par le seigneur pour rachat du droit de quint ${ }^{45}$. Dalhousie, en 1826, proclame ces dispositions libérales; elles devraient délivrer le pays...... Or le pays reste sourd; presque personne ne change de tenure ${ }^{46}$.

La métropole ne se résigne pas à l'échec; elle en veut connaître les raisons et supplie le gouverneur de lui dire enfin quelles mesures elle doit voter pour faire disparaître cet odieux vestige du régime français. Kempt, en 1830, entreprend de répondre et s'acquitte magistraloment de sa tâche. Le gouverneur écrit en substance: le véritable obstacle à la conversion des tenures ne consiste pas dans l'insuffisance des lois et des instructions, mais dans l'indifférence générale de la population: censitaires et seigneurs. L'habitant n'est pas disposé à convertir la tenure de sa terre, parce qu'il est attaché aux coutumes

41. Ellice a W. Horton, 15 avril 1823, 15 octobre 1824, APC, Q175:60, 46.

42. Gazette de Quebec, 9 février 1824, APC, Collection Dalhousie, 8.

43. W. B. Munro, Documents relating to the Seigniorial Tenure in Canada, 15981854, Toronto $1908,347$.

44. 6 Geo IV, cap. 59, i-vii, APC, Q175:1-31.

45. Bathurst à Dalhousie, 12 aout 1825, APC, G14:98.

46. Dalhousie à Bathurst, 19 juin 1826, APC, Collection Dalhousie, 11. 
françaises et surtout parce que les charges seigneuriales sont actuellement si légères que personne ne les considère comme un fardeau dont il vaut la peine de se défaire par la mutation des tenures. Les terres sont toutes concédées à des taux très bas de cens et rentes; le droit de mouture du moulin banal est moins élevé que celui qu'on paie aux moulins des cantons; la vente des fermes arrive si rarement que même les lods et ventes constituent à peine pour les campagnes un fardeau perceptible. Bien que ces redevances soient peu sinties du censitaire, leur disparition dans les seigneuries peuplées priverait le seigneur d'une grande partie de ses revenus. La conversion des tenures serait pour ce seigneur une mauvaise affaire, car elle se ferait sans doute à bas prix, à l'exemple de la Couronne qui n'exige que $5 \%$. Seuls auront avantage à changer de tenures, les quelques seigneurs possesseurs de fiefs non peuplés ou à peine peuplés, car ils deviendraient alors, à bon compte, propriétaires absolus de vastes domaines. Et Kəmpt de conclure: il ne faut s'attendre à des demandes de conversion de tenures que de ces quelques seigneurs et des bourgeois des villes où les lods et ventes sont de sérieux inconvénients; les décrets n'y peuvent rien; l'abolition du régime seigneurial n'aura lieu que dans un Bas-Canada plus avancé " en richess?, en population et en intelligence " ${ }^{4}$.

$K$ ?mpt l'avait donc clairement compris: toute cette agitation pour supprimer la tenure seigneuriale ne venait ni des censitaires canadiens, ni de la grande majorité des seigneurs dont les fiefs étaient colonisés, mais ne regardait que le petit groupe de seigneurs britanniques dont les terres étaient encore vacantes vers $1820-1830$ et qui pouvaient, une fois la conversion opérée, exploiter le bois à leur guise ou attirer les immigrants britanniques. Les rares mutations demandées ou obtenues par les seigneurs et les bourgeois, entre 1825 et 1842, prouvent que Kempt avait été bon prophète ${ }^{48}$.

Mais poursuivons.

Pour mettre fin aux doutes entretenus dans la colonie, la loi impérialo de 1825 stipulait en outre que les terres tenues en soccage, de même que les terres passées à cette catégorie (les terres seigneuriales

47. Kempt a G. Murray, 12 janvier 1830, APC, Q193-1:109-17.

48. Report (on the) Seigniorial Tenure, Kingston 1844, 69. 
commuées) seraient soumises aux lois anglaises pour la façon de donner, de vendre et d'hériter ${ }^{49}$. N'était-ce pas fournir aux Canadiens une raison de plus de tenir a u régime seigneurial? En effet l'Assemblée proteste avec véhémence. Cette introduction des lois civiles anglaises lui apparaît une violation des "constitutions") de 1774 et de 1791 . Elle demande à Londres le rappel des lois impériales de 1822 et de $1825^{5}$. L'Assemblée semblait donc attacher beaucoup d'importance à cette intrusion de lois civiles anglaises; elle en faisait son principal grief contre les tentatives de remplacer la tenure seigneuriale. Kempt cependant avertit Londres que cette complication d'ordre légal détourne les Canadiens de la conversion des tenures, mais que ce n'est pas leur seule objection. Sans elle, l'indisposition générale contre le changement de tenures subsisterait quand mêm $\vartheta^{51}$.

Le même gouverneur avait dit pourquoi les Canadiens ne transformeraient pas la tenure des terres qu'ils possédaient déjà. A son tour, Aylmer exposera l'attitude des Canadiens envers le genre de tenure qu'ils désirent pour les nouvelles terres à concéder. Lorsqu'on offre en vente les terres de la Couronne, a-t-on dit à Aylmer, on devrait permettre aux acheteurs de choisir entre la tenure seigneuriale et la tenure en soccage. Mais il y a doute qu'on puisse agir ainsi, car les lois de 1822 et de 1825 indiquent que l'intention de Londres est d'abolir le régime seigneurial et cette politique serait contrecarrée par de nouvelles concessions seigneuriales. Néanmoins, Aylmer soumet le problème à Londres et plaide la cause des Canadiens: les habitants sont très attachés à la tenure seigneuriale; leur refuser le droit d'acquérir des terres de la Couronne selon cette tenure équivaut à les exclure de tout achat de terres de la Couronne; au contraire, la possibilité d'avoir des terres selon la tenure seigneuriale serait très bien vue des Canadiens. Aylmer demande donc la permission de concéder selon les deux tenures, au choix du concessionnaire ${ }^{52}$. D'ailleurs, cette demande ne fait que corroborer l'enquête impériale de 1828; n'y

49. 6 Geo IV, cap. 59, viii, APC, Q175:1-31. 529-33.

50. Adresse de la Chambre d'Assemblée au Roi, 18 mars 1826, APC, Q176-2 :

51. Kempt à G. Murray, 12 janvier 1830, APC, Q193-1:116-7.

52. Aylmer à G. Murray, 19 décembre 1830, APC, Q195-2:272-4. 
conseillait-on pas de créer d'autres seigneuries ${ }^{5}{ }^{3}$ ? Ainsi, vers 1830, soixante-dix ans après la Conquête, la masse des Canadiens restait attachée à la tenure seigneuriale et espérait en de nouvelles seigneuries.

Londres refuse la permission réclamée par Aylmer, parce que contraire à l'esprit des lois de 1822 et de 1825 . Et trompé par la protestation de l'Assemblée de mars 1826, protestation qui visait uniquement l'introduction des lois civiles anglaises, le gouvernement impérial répond au gouverneur: il n'est pas nécessaire da concéder selon le mode seigneurial et les Canadiens n'auront plus aucune objection au soccage puisque bientốt une loi impériale donnera à la Législature coloniale le pouvoir de déterminer quelles lois civiles s'appliquent aux terres tenues en soccage ${ }^{54}$.

Or voici qu'un seigneur canadien (Hertel de Rouville) demande une seigneurie de huit lieues de profondeur; il y veut établir les nombreux fils de cultivateurs du district de Québec, qui sont sans terre mais ne veulent ni du soccage ni des cantons ${ }^{55}$. Londres, on pouvait s'y attendre, rejette cette pétition qui lui semble une monstrueuse absurdité: se peut-il que des gens refusent de s'établir sur des terres parce qu'elles sont exemptes de charges féodales? Ou plutôt, Londres croit découvrir, derrière cette attitude des Canadiens, une tentative de conserver un organisme qui empêche les immigrants de coloniser le Bas-Canada..... Et l'on en vient à se dire que le temps est passé d'obéir à de tels caprices au préjudice de l'épanouissement de l'Amérique du Nord britannique ${ }^{56}$.

Avec 1831 prend donc fin officiellement, pour les autorités locales, le pouvoir de concéder de nouvelles seigneuries. 1791 avait déjà marqué la fin pratique de ce pouvoir. Du reste, ressuscité en 1771 , il n'avait jamais été exercé en faveur des Canadiens.

LUTte NATIONALE: LOIS ET TENURE.

On ne saurait isoler la question seigneuriale de la lutte nationale entre Canadiens et Britanniques pour la possession du Bas-Canada.

53. Garneau, Histoire du Canada, 8:123.

54. Goderich à Aylmer, 13 mars 1831, APC, G22:290.

55. J.-B.-R. Hertel de Rouville au Roi, 8 mars 1831, APC, Q198-1:97-8.

56. Goderich à Aylmer, 22 février 1832, APC, G24:166. Et commentaires initiales J.S., APC, Q198-1:93-6. 
L'Assemblée, dominée par les Canadiens, exige le rappel de la législation impériale de 1825 parce que celle-ci introduit dans la province les lois civiles anglaises ${ }^{57}$. Le Conseil législatif, dominé par les Britanniques, s'oppose au rappel de cette même législation parce que celle-ci a le mérite de libérer les cantons des lois civiles françaises, obstacle à la colonisation de ces cantons par les immigrants venant du Royaume-Uni ${ }^{58}$. Londres, en 1831, donne à la Législature du BasCanada (Assemblée et Conseil législatif) la permission d'amender la loi impériale de 1825 et de fixer quelles lois civiles conviennent le mieux aux terres tenues en soccage ${ }^{59}$. Natur le Conseil ne purent s'entendre et se chamaillèrent de plus belle ${ }^{60}$.

Encore que l'Assemblée insiste beaucoup sur la question loi, elle ne laisse pas de protester, et ce, encore vers 1830-1832, contre une conversion des tenures qui priverait le cultivateur canadien du droit d'acquérir un lot aux taux ordinaires. Elle veut que l'habitant conserve au moins tous ses droits quant à la façon d'obtenir une terre, même dans les seigneuries qui seraient reconcédées en soccage. Encore et toujours les autorités s'y objectent ${ }^{6}{ }^{1}$.

En 1834, dans les Quatre-vingt-douze Résolutions, l'Assemblée revient à la charge. Si la conversion des tenures, fait-elle de nouveau remarquer, s'opérait selon la loi impériale de 1825, les seigneurs deviendraient maîtres absolus des domaines non encore habités et les cultivateurs y perdraient lours privilèges d'obtenir des terres aux taux traditionnels. Les Canadiens ne se refusent pas à modifier la tenure seigneuriale; mais ils entendent que ce soit la législature locale et non le parlement impérial qui règle cette question. Puis, l'Assemblée dénonce le vice capital de la mesure votée par Londres en 1825, laquelle vise à introduire de force les lois civiles anglaises. Cette loi impériale qui est une loi de violence, doit être rappelée ${ }^{62}$.

57. Pétition de l'Assemblée, 26 mars 1831, APC, Q197-2:297-8.

58. Résolution du Conseil lég., 31 mars 1831, APC, Q197-2:299-300.

59. 1 Will IV, cap. 20, APC, G22:347.

60. Munro, The Seigniorial System in Canada, 229-30.

61. Ibid., 230-1.

62. Résolutions 57-62. 
En 1837, le gouvern?ment métropolitain fait savoir qu'il consentira à l'abrogation du Canada Tenures Act de 1825, mais pas avant que la législature du Bas-Canada n'ait elle-même aboli les charges seigneuriales et déterminé quelles lois civiles régiront les terres en soccage ${ }^{63}$. Pas plus en 1837 qu'en 1831, l'entente n'était possible entre l'Assemblée et le Conseil législatif. Il faudra la suppression de la législature du Bas-Canada et un " esprit nouveau " pour en arriver à une solution.

\section{LiB ÉRAUX ET BOURGEOIS CONTRE LA TENURE}

En 1837 un esprit libéral à la mode française anime quelques meneurs de l'insurrection. Pour exciter les paysans à la résistance, certains leur promettent de les débarrasser des seigneurs, des corvées, des rentes...... ${ }^{64}$ Des abus, il faut le diro, prêtaient à ces déclamations. Le peuple désirait la réforme du régime et c'était bonne tactique révolutionnaire que d'exploiter et d'amplifier les griefs de la masse. Les meneurs avaient particulièremənt beau jeu dans la vallée du Richelieu où plusieurs seigneurs d'origine britannique se montraient assez exigeants. Durham fait grand cas de cette poussée doctrinale de 1837-38. Il paraît attribuer aux seuls députés dominés par leurs passions nationalistes et conduits par quelques seigneurs réactionnaires, le maintien d'une féodalité dont les paysans canadiens n'auraient pas voulu ${ }^{65}$. Durham a tort de généraliser en s'appuyant sur le cri libéral de 37, et sur quelques pétitions qui portent surtout la signature de Britanniques. Trop de faits depuis 1760 ont prouvé l'attachement des paysans au mode seigneurial d'acquérir des terres. Au reste Durham ne peut s'empêcher de le reconnaître ailleurs: " la.tenure féodale fut estimée comme un procédé facile et adéquat de coloniser un jeune pays " ${ }^{66}$.

Jusqu'ici ce sont des seigneurs (de Lanaudière, Caldwell, Ellice) et ce sont des gens tels que Sewell, Milnes, etc., qui ont déclenché les attaques contre la tenure seigneuriale. Les uns et les autres voulaient, par l'abolition de ce régime, faciliter aux Britanniques la colonisation

63. J. Russell, Sixième Résolution, 6 mars 1837, RAPC, (1931), 427-28.

64. J.-B. Dupuy, notaire, 12 juillet 1842; F.-B. Ricard, ptre, 10 juin 1842; pièces jointes au Report (on the) Seigniorial Tenure, (Kingston 1844), 85, 82.

65. C. P. Lucas, Lord Durham's Report on the affairs of British North America, 3 vols, Oxford 1912. 2:24-5.

66. Ibid., 50. 
surtout agricole du Bas-Canada. Après l'Union des deux Canadas, l'offensive provient d'autres quartiers. Ce ne sont plus des seigneurs, mais des hommes d'affaires (Britanniques en tête) qui entrent en lice, en vue de faciliter la colonisation surtout ind'ustrielle et commerciale du Canada-Est. Nous assistons à la lutte du bourgeois contre le seigneur et où l'on s'applique à manœuvrer le paysan.

Dès l'ouverture du parlement du Canada-Uni, on demande une enquête. ${ }^{67}$ En 1842, Bagot nomme, à titre de commissaires-enquêteurs, deux Britanniques et un Canadien. Ceux-ci adressent aux censitaires et aux seigneurs de longs questionnaires, amassent une documentation incomplète et bâclent leur rapport pour octobre $1843 .{ }^{68}$ La lecture des pièces annexées au rapport ne révèle chez les censitaires aucune attitude nouvelle. La plupart des censitaires des vieilles seigneuries, sans se priver de noter les abus commis par quelques seigneurs, n'insistent aucunement pour une abolition du régime, mais en désirent la réforme par un retour aux anciens taux de cens et rentes et par l'application des lods et ventes au fond de terre seulement. L'abolition, seuls la prônent, un petit nombre de censitaires (Britanniques surtout qui proclament leur aversion en termes fort expressifs) et quelques Canadiens. Tous s'accordent sur un point: le caractère onéreux des lods et ventes, bien plus onéreux toutefois pour les villes et les villages que pour les campagnes. On ne craint pas de le dire: c'est un vol; le seigneur n'a pas droit au douzième du prix de vente quand la valeur de la propriété dépend avant tout des constructions et des améliorations du censitaire et non du fond de terre ou de l'emplacement concédé par le seigneur.

Les seigneurs répondent de mauvais gré à l'enquête; ils se sentent menacés de confiscation. D'après eux, dans les régions peuplées depuis longtemps, les taux de cens et rentes sont demeurés très bas. Ces taux, même augmentés dans les dernières concessions, restent encore nimimes et sont préférables à l'achat de terres dans les cantons. Des seigneurs, des notaires et des prêtres font remarquer, avec raison, la pauvreté générale du paysan canadien, accablé d'une série de mauvaises

67. Résolutions de l'Assemblée législative, 7 septembre 1841, ibid., 20-1.

68. Report of the Commissioners appointed to enquire into the state of the laws and other circumstances connected with the seigniorial tenue in Lower-Canada, and appendix, 4 octobre 1843, Kingston 1844. 
récoltes, incapable de débourser le capital nécessaire pour racheter brusquement les charges seigneuriales. Dans la crainte d'être ruiné par une telle opération, le censitaire canadien ne désire pas l'abolition du régime, à moins que l'on n'entende par là : confiscation des droits des seigneurs.

En juge-t-on par les sources qui ont dû servir à l'élaborer, le rapport de 1843 a de quoi déconcerter. On s'attendrait à trouver un document nuancé, un exposé loyal des diverses opinions. Au contraire, ce rapport est un pamphlet, une charge contre le régime seigneurial et les seigneurs. On veut bien concéder l'utilité du régime aux premiers temps de la colonie. Mais depuis 1760 , les seigneurs, protégés, par les cours de justice, auraient doublé, triplé, quadruplé, quintuplé, sextuplé même les taux de cens et rentes, multiplié les corvées, raflé le bois, refusé de concéder ou vendu à la dérobée par l'entremise d'agents, etc... si bien que le régime, tel qu'évolué, écraserait le cultivateur et, par les iniques lods et ventes, déprécierait la propriété, taxerait et paralyserait l'essor de la colonie. Bref, on ne saurait inventer un meilleur système pour tenir un homme en un perpétuel esclavage. A tout prendre, un reliquat d'un âge barbare. Et les commissaires-enquêteurs, sans envisager la réforme du régime, se prononcent catégoriquement pour la conversion des tenures. Que l'on abolisse le régime, et la prospérité s'épanouira au pays et la liberté aiguillonnera les énergies. Que le régime subsiste, et la population végétera sans espoir.

Le rapport de 1843 est donc en tout point conforme à ce que les Britanniques pensaient de la tenure "féodale " depuis le jour où, avec les premiers Loyalistes, ils ont voulu coloniser leur conquête. Finiront-ils par l'emporter?

La loi impériale de 1825 ne permettait au censitaire de changer la tenure de sa terre que si d'abord le seigneur avait converti la tenure de sa seigneurie. Comme trois seigneuries seulement remplissaient cette condition, très peu de censitaires auraient pu se libérer des charges seigneuriales. On s'imagina donc, en 1845, qu'il suffirait de donner la liberté au censitaire de changer la tenure de sa terre, même dans le cas où son seigneur n'aurait pas transformé la tenure de sa seigneurie, pour voir immédiatement tous les habitants se lancer dans un vaste mouvement d'émancipation. Bien mieux, pour faciliter ce mouvement, on décréta que la conversion se ferait en franc alleu roturier, selon les lois civiles françaises, et non en soccage selon les lois anglaises. Encore 
une fois, et contrairement à l'attente des " abolitionnistes ", le pays ne répondit pas. ${ }^{6} 9 \mathrm{Il}$ fallut en venir à la conversion dite obligatoire par une loi votée en 1854. Évaluées par des arbitres, les charges seigneuriales furent transformées obligatoirement en une rente donstituée payable chaque année par le censitaire, mais aussi rachetable. ${ }^{70}$

Cependant,-et c'était à prévoir - les paysans canadiens ne purent et ne voulurent pas, pour la plupart, racheter cette rente. Néanmoins les promoteurs de l'abolition avaient obtenu ce qu'ils recherchaient.

\section{CONCLUSION-UN COMPROMIS}

La façon dont on disposa de la question seigneuriale au milieu du XIXe siècle, apparaît comme un compromis entre les Britanniques et les Canadiens, entre les paysans et les bourgeois. Les Britanniques se libèrent d'une tenure abhorrée dont ils souhaitaient la disparition depuis 1785. Ils n'en doivent pas moins abandonner leurs rêves de $1790,1801,1816,1825$, qui étaient de peupler les seigneuries de colons britanniques. Les Canadiens consentent à la suppression d'un régime qu'ils avaient longtemps deffendu; mais ils gagnent de garder aux terres seigneuriales commuées les lois civiles françaises. D'ailleurs, même avant 1837, les dirigeants canadiens admettaient qu'il faudrait, un jour, réformer le régime, au moins au sujet des lods et ventes. ${ }^{71}$ Les paysans, d'une part, auraient accepté la réforme et la continuation du régime. D'autre part, tout en regrettant de ne pouvoir obtenir de nouvelles seigneuries dans les centres de colonisation agricole, (car, pour des colons, acheter des terres selon la méthode utilisée dans les cantons n'avait pas cessé de paraître plus compliquée que de payer annuellement une rente) les habitants des vieilles seigneuries où l'on avait dépassé depuis longtemps le rude stage des premiers défrichements, ne s'opposaient aucunement à la suppression des charges seigneuriales, pourvu que le procédé ne comportât rien d'onéreux. Onéreux, le procédé choisi en 1854 ne l'était pas. L'habitant n'était

69. Munro, The Seigniorial System in Canada, 242-3.

70. The Seigniorial Tenures Act, 18 décembre 1854, dans Kennedy, Statutes, Treaties and Documents, 519-32.

71. First Report of the Committee of the House of Assembly (on the) Crown Lands, Quebec 1821, 8. Lucas, Lord Durham's Report, III, 50. 
tenu à aucun déboursé obligatoire et immédiat; il pouvait continuer à payer une rente, avec la faculté de se libérer à volonté.

Du même coup, les bourgeois, les hommes d'affaires se débarrassent des lods et ventes qui, bien plus que les cens et rentes, ont déterminé I'abolition du régime. Ils obtiennent un droit absolu de propriété et non plus cette propriété en quelque sorte conjointe du seigneur et du censitaire. Droit absolu de propriété devenu nécessaire en l'ère nouvelle qui s'ouvrait pour l'Amérique du Nord britannique, vers 1850, avec la fin du pacte colonial et avec l'essor général de la vie économique depuis l'Union des Canadas. Le régime seigneurial et l'agriculture paysanne canadienne ne s'opposaient pas jusqu'à incompatibilité; mais ce régime ne pouvait coexister avec la Révolution industrielle. Le système avait été organisé au XVIIe siècle pour promouvoir la colonisation agricole. Or la vallée du S.-Laurent était devenue le chenal d'un intense commerce; elle allait se transformer, dans ses divers centres, en une région d'industrie. On ne pouvait donc supporter ce système. Son existence est qualifiée de public evil, non toutefois par la masse des paysans, mais par la classe commerciale et industrielle. Et sa suppression n'est pas l'œuvre des paysans et ne vise pas à favoriser d'abord les paysans. L'abolition incomplète du régime seigneurial, vers 1850 , relève de l'histoire de l'industrie et du commerce bien plus que de l'histoire de l'agriculture.

Le rôle primordial de la bourgeoisie, la raison profonde de l'abolition du régime et l'évolution de la pensée des dirigeants canadiens sur ce problème sont clairement exposés par J.-C. Taché:

Jusqu'à ce jour, je ne m'étais occupé de la Tenure Seigneuriale qu'au point de vue du redressement des griefs et ne voulais de commutation que ce qu'il en fallait pour soustraire l'industrie à l'entrave que lui met la Tenure en Censive dans certains cas. En cela, je ne faisais qu'adopter l'opinion des hommes les plus éminents qui se sont occupés sérieusement de cette question et obtempérer aux vœux exprimés par la grande majorité des censitaires dans les requêtes par eux présentées aux autorités.

Je suis encore d'opinion que pour un Pays nouveau et pour un Pays exclusivement agricole, il n'est pas de meilleure manière d'octroyer les terres que la manière voulue par la Tenure Seigneuriale, telle que constituée en Canada par les lois françaises. ${ }^{72}$

72. J.-C. Taché, De la Tenure Seigneuriale en Canada et projet de commutation, Québec 1854, 3. 
Mais Taché en est arrivé à prôner l'abolition du régime " comme une mesure d'utilité générale, nécessitée par un changement survenu dans la manière d'être de la société... " Et voici comment il s'explique:

Je citerai un exemple duquel je ferai ressortir un fait important dans la cause. La province en commun avec de puissantes compagnies est engagée dans la confection d'un réseau de voies ferrées; mais il faut traverser le sol seigneurial et en acquérir une portion; or chaque parcelle du sol féodal, en vertu de l'indivisibilité du cens, est soumise au droit de Lods et Ventes; qu'une transaction arrive équipolant à Vente et il faudra payer aux Seigneurs la douzième partie de la valeur des chemins de fer. Dans ce cas, comme dans celui de la fondation ou de l'agrandissement des villes ou de l'etablissement d'usines ou manufactures, ce n'est pas le Censitaire détenteur actuel de la propriété rurale qui souffre le mal le plus grand; mais l'industriel; mais le capitaliste, et par contre-coup la sociêté entière, dont les progrès peuvent se trouver retardés, paralisés même. ${ }^{73}$

Maurice SÉguin

Licencié ès lettres

de l'Université de Montréal

73. Ibid., 5-6. 